\title{
Recommendation Systems for E-Commerce: A Review
}

\author{
Priya $S^{1}$, Mansoor Hussain $D^{2}$ \\ Student, CSE, SKCET, Coimbatore, India ${ }^{1}$ \\ Assistant Professor, CSE, SKCET, Coimbatore, India ${ }^{2}$
}

\begin{abstract}
Recommendation as a social process plays ansignificant role wherepeople rely on external knowledge to make decisions about an artifact of interest. Recommendation system is an intelligent system that generates a ranked list of items on which a user might be interested. Nowadays, there is a big variety of different approaches and algorithms for data filtering and recommendation giving.Recommendation techniques can be classified into three major divisions: Collaborative Filtering, Content Based and Hybrid Recommendations.This paper compares and elaborates these approaches and discusses their limitations by describing the problems suffered by recommendation techniques.
\end{abstract}

Keywords: Recommendation system, Content-Based Recommendation, collaborative filtering, coldstart, scalablility.

\section{INTRODUCTION}

Recommender systemsbecome an importantresearch area since the appearance of the first papers on collaborative filtering in 1990s. There has been much work done both in the industry and academic area for developing new approaches in recommendersystems over the last decade. The interest in recommendation system still remains high because it constitutes a problem-rich research area and because of practical applications that help users to deal with information overload and provide personalized recommendations, content, and services to them [6]. Examples of such applications include recommendationsof variousbooks, CDs, products at Flipkart.com, movies by MovieLens, and news at VERSIFI Technologies.Recommender system is one application which is being used by many vectors and online service providers to believe the necessity of online users.. Thus the recommender system is presented as an intelligent system, which identifies the user category having a basis on the user information and then user interest analysis. Once such information is obtained, in second stage, the analysis is performed to obtain the similarity group respective to necessity products and services.To perform such kind of analysis there are some existing techniques such as content based as well as collaborative recommender systems [7].This paper is structured as follows.The summary of the related work of recommendation system is explained in section II. This is followed by a detailed description of three recommendation techniques in section III. Comparison of algorithms and recommenders are provided in the section IV.Then the comparative analysis of three recommendation systems is provided in section V. Section VI concludes with suggesting the extension of proposed work.

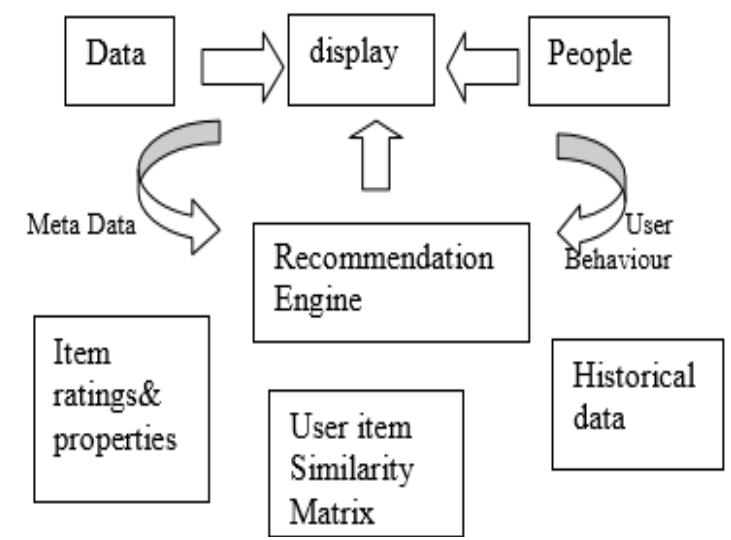

Fig 1[8]: Architecture of Recommendation system

\section{RELATED WORK}

Recommendation system has been so extensively used these days that it has become a preferable choice for researchers. First paper on recommender system have been explained to increase the reliability of recommendation system. In year 
2005 John O'Donovan, Barry Smyth have taken trust of users as the percentage of correct predictions that a profile has made in general or with respect to a particular item. In year 2007 Paul Resnick proposed an idea of "influence limiter algorithm" in recommender system. Which prevents any attack that are irrelevant result for the search and limits the number of content that an attacker can modify. Description of the design of a recommender system uses knowledge stored in the form of ontologies. The interactions amongst the peer agents for generating recommendations are based on the trust network. Recommendations about a product given by peer agents are in the form of Intuitionistic Fuzzy Sets specified using degree of membershipand uncertainty.In 2008KleanthiLakiotaki, SteliosTsafarakis, and NikolaosMatsatsinis proposed UTA-Rec that incorporates Multiple Criteria Analysis methodologies. The system's performance and ability of addressing certain shortfalls of existing Recommender Systems is demonstrated in the case of movie recommendations, product recommendation[7].

\section{RECOMMENDATION SYTEM TECHNIQUES}

Recommender systems are a powerful information filtering technology used to either predict whether a particular user will like a particular item or to identify a set $\mathrm{N}$ items that will be of interest to a certain user.In this section, we describe three recommendation systems, such as content based System, collaborative filtering, Hybrid System

\section{A. Contentbased System}

Content based recommendation [3] systems recommends an item to a user based upon a representation of the itemand items common to those that user are previously purchased or reviewed [2]The user will get recommendation similar to the ones whichthe user preferred in the past. Such systems used in recommending web pages, TV programs and news articles, advertisements etc. All content based recommender systems has few similar thingslike description of items, user profiles and techniques to compare profile to items to identify which is the required recommendation for a particular user. A profile of the user has information about a user and preferences. Taste depends on how the items are rated in the earlier log. While preparing a profile, recommender systems setup a survey, to get beginning information about a user in order to avoid the new-user problem.In the recommendation process, the engine compares the items that were rated positively by the user with the items unrated items that looks for similarities. Those unrated itemsthat are mostly similar to the positively rated ones will be provided to the user[5].Content-Based systems make suggestions upon item factors and user interest profiles. Likewise, personalized profiles are created automatically

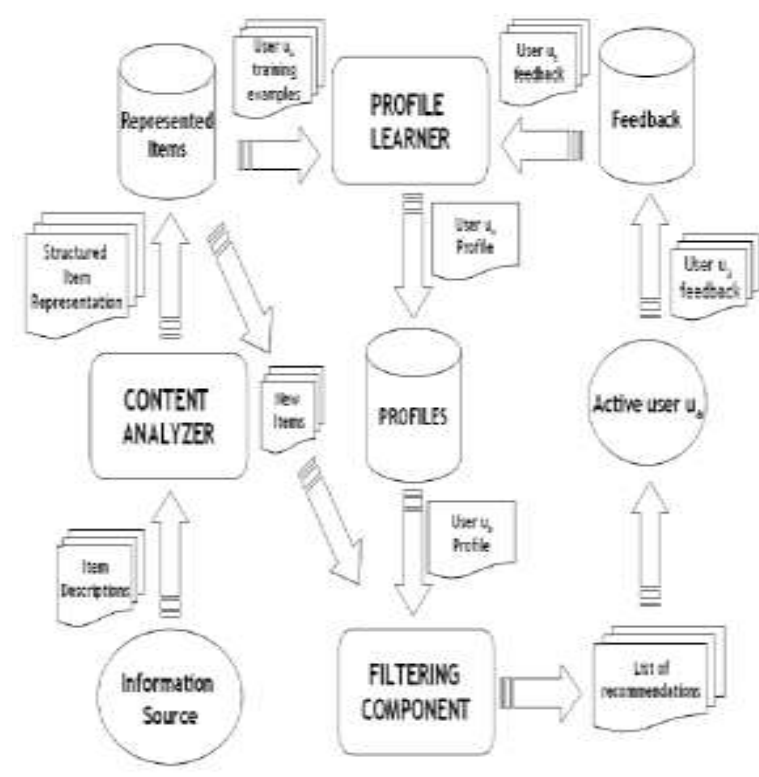

Fig. 2[19]. Content based Recommendation System

through user feedback, and describe the type of items a person likes. In order to estimate what items to recommend, collected user information is compared against content features of the items to examine.For example[1], in movie recommendation, in order to recommend movies to user $\mathrm{c}$, the content-based recommender system tries to understand the commonalities among the movies user $\mathrm{c}$ has rated more in the past (specific actors,actress, directors, genres, subject matter, etc.).Finally, the movies that have a high degree of similarity to whatever the user's preferences are would be recommended.System has a huge amount of database that consist of the items to be recommended and the features of these items and it is termed as Item Profile.The users provide some sort of information about their 
preferences to therecommender system. Combining the item information with user preferences, the systembuilds a profile of the users.

\section{B.Collaborative Filtering}

A CF-based system[10] associates a user with a group of like-minded users based onuser interaction information over all the items, and then recommends to the user the item enjoyed by the group. User interaction information might be provided explicitly by the individual person or gathered implicitly by a software agent.1)Explicit user information collection basically depends on personal input by the user. A common feedback technique is that allows users to express their opinions.2) Implicit feedback does not require any additional interaction by the user during the process of constructing profiles and automatically updates as the user interacts with the system.A group of likeminded users are said to be neighbours. The basic assumption is that the users with similar behaviour on observed items (e.g., ratings) will have similar tastes on unobserved items [6]. In the beginning, Goldberg et al used Collaborative filtering (CF) for introducing their filtering system that gives ability to customer for explanation their e-mails and documents [4]. The task of recommender algorithm[2] concerns the prediction of the user's rating for the target item that the user has rated, based on the users' ratings on observed items.

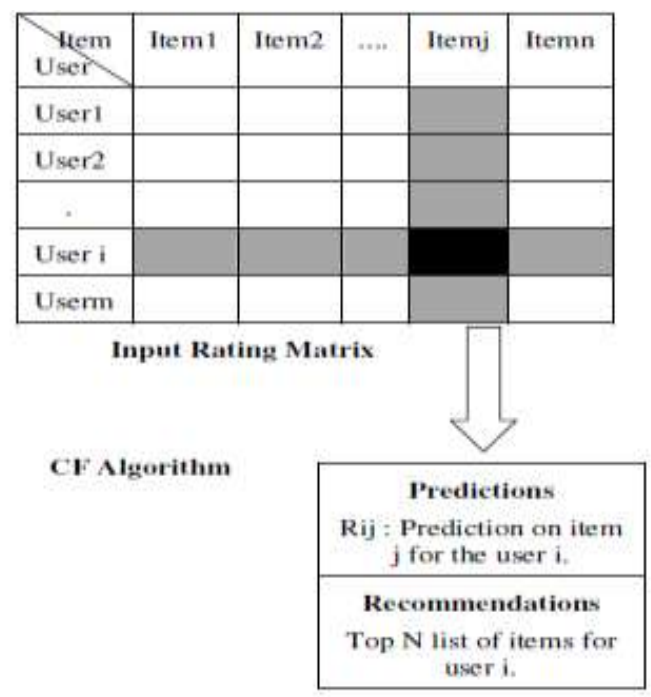

Fig 3[2].Collaborative Filtering Process

$\mathrm{CF}$ algorithms represent entire user-item space as a rating matrix ' $\mathrm{R}$ '. Each entry in the Rij in matrix represents the preference score (rating) if the ith user on the jth item. Each individual rating is within a numerical scale and it can be 0 as well, providing that user has not yet rated this item. CF problemalso includes the prediction of rating for the unrated item. For the estimation of rating, similarities between items and users are predicted using different approaches. Thus, the two related problems refers finding set of $\mathrm{K}$ users that are most similar to a given user and finding set of $\mathrm{K}$ items that are more similar to a given item. Finally with these similarities, recommendations that are produced at output interface can be of twotypes: Prediction and Recommendation. 1) Prediction is an numerical value, Rij, that the predicted score of item $\mathrm{j}$ for the user $\mathrm{i}$. The predicted value is within the same scale which is used by all users for rating. Recommendation is a list of top $\mathrm{N}$ items that the userlikes the most. Thus the output interface is called as TopNrecommendation. Collaborative filtering [4 ]is usually classified into two parts 1)Model-based CF , provide recommendations by estimating parameters of statistical models for user ratings to make a prediction.2) Memory-based $\mathrm{CF}$, uses the entire or a some of the user-item database to generate aprediction.

\section{C.Hybrid Recommendation}

Hybrid Recommendation is a combination of both collaborative filtering and content based approach .With Hybrid Recommendation many types of problems such as Cold-Start problem can be handled using the hybrid recommendations .Different ways of hybridization are implementing CF and CB separately and joining their predictions,Incorporating some content based methods into collaborative filtering ,Incorporating some of the collaborative characteristics into content based approach, Constructing a unifying model that posses both content-based and collaborative characteristics[2].Many combination approaches[4] that are used for building hybrid recommendation systems are as follows:

A)Mixed: This method point to the suggestions and recommendations from a setof variousrecommendation systems, that are presented simultaneously. 
B)Weighted: Produces only

single recommendation by using of the votes and rates that are created by some recommendation approaches.

C)Feature combination: The characteristics that relate to various recommendation data resources are assembled into a single recommendation system algorithm.

D)Cascade: One of the recommendation systems corrects the prompts and recommendations that are presented by another recommendation system.

E)Featureaugmentation: Outputi.e results from one approach are employed as input data and characteristics for another recommendation method.

F)Meta level: The approach that is established by one recommendation system is utilized as a input for another approach.

F)Switching: In this method, recommendation system shifts among different recommendation approaches acceding to the current situation .

\section{COMPARISONS BETWEEN ALGORITHMS AND RECOMMENDERS}

Recommender systems are widely used in many domains and especially popular for recommending books, music, movies and TV programs among others. Amazon.com uses item-based collaborative filtering (CF) to predict the items which is suited best from the enormous set of potentially interesting items.TiVo Suggestions uses a combination of item- based collaborative filtering base their recommendations on like-minded users and Bayesian content based filtering use features from the items themselves (like genre, cast, age of the show) valued by the user, to generate recommendations. Similarly some websites such as Jester 2.0, LIibra, Group Lens, MeeVee, Ringouse different recommendation types and algorithm. Thus the memory based algorithms are mostly preferred by commercial recommenders and correlation algorithms are required when there is sparse data.

\section{TABLE I[20] COMPARISON AMONG THE ALGORITHMS AND RECOMMENDERS}

\begin{tabular}{|l|l|l|}
\hline Recommender System & Type & Algorithm \\
\hline Amazon & Collaborative & Item based collaborative filtering \\
\hline TiVo & Hybrid & $\begin{array}{l}\text { Item-based collaborative filtering, } \\
\text { Bayesian content based filtering }\end{array}$ \\
\hline Jester 2.0 & Collaborative & Cluster \\
\hline Libra & Hybrid & Bayesian Learning algorithm \\
\hline Group Lens & Hybrid & Correlation \\
\hline
\end{tabular}

\section{THE ANALYSIS OF RECOMMENDER SYSTEM}

In this section, the Recommender approaches (i.e., content-based, collaborative filtering and hybrid filtering) are analysed.Recommender-systems research mainly depends on evaluations to assess the effectiveness of recommendation approaches. The usefulness of the evaluation [9], the re-implementation of the approach, and the reproducibility and duplication of the results is guaranteed. The discussed recommendation approaches categorized as being content-based, collaborative, and hybrid on rating estimation have performed good in several applications, such as for recommending books, CDs, and news articles, etc. For example, for movie recommendation application, by increasing the traditional memory-based collaborative filtering approach to take into attention the contextual information, such as when, where, and with whom a movie is seen, the resulting recommender system could overtake the pure traditional collaborative filtering method to provide better recommendation [1] Therefore, there is a need to grow more advanced recommendation methods is even more required for such types of applications.

TABLE II [3] COMPARISON AMONG THREE RECOMMENDATION SYSTEMS

\begin{tabular}{|l|l|l|}
\hline Types & Advantages & Disadvantages \\
\hline Content Based & User independence Transparency & Limited content analysis. \\
& Quality improved over time. & Over-specialization and serendipity. \\
\hline Collaborative & $\begin{array}{l}\text { Easy to create and use. } \\
\text { New data can be added easily. } \\
\text { More applicable . }\end{array}$ & $\begin{array}{l}\text { It totally depends on human ratings. } \\
\text { Sparsity leads to Insufficient Data. } \\
\text { Cold start problem. }\end{array}$ \\
\hline Hybrid & $\begin{array}{l}\text { Improves user preferences. } \\
\text { Avoids cold start problem. } \\
\text { No sparsityproblem. }\end{array}$ & Difficult to develop a trust network. \\
& \\
\hline
\end{tabular}




\section{CONCLUSION}

Recommendation system are anefficient technology that help people to find their interests with less effort,less work and less spending time with more accuracy .This paper explained about the three recommendation system. Thus these recommendation systems have offered many methods for searching and filtering information.Recommender system are rapidly becoming a important tool in E-commerce on the Web and Movie Websites.The improved modelling of users and items, incorporation of the contextual information into the recommendation process, support for multicriteria ratings, and facility of a more flexible and less interfering recommendation process. In future,enhanced clustering algorithms as well as better prediction generation schemes which is used to improve prediction quality for e-commerce have to developed.

\section{REFERENCES}

[1] G. Adomavicius and A. Tuzhilin." Toward the next generation of recommender systems: A survey of the state-of-the-art and possible extensions". IEEE transactions on knowledge and dataengineering, pages 734-749, 2005.

[2]M sharma,Smann ,"A survey of Recommender systems :Approaches and Limitations ”,2013.

[3] R pagare ,Ashinde," A study of recommender systems Techniques",2012

[4] A .H Rafsanjani,NSalim,A R Aghdam,K B Fard,'Recommendation system: a Review",2013[5]D Asanov,"Algorithms and Methods in recommendation systems ",2012

[6] G..Adomavicius, A Tuzhilin," Recommendation Technologies:Survey of Current Methods and Possible Extensions",2004

[7] Parul ,k .khanna,'Literature Survey: Recommender Systems”, March 2015, Volume 3 Special Issue, ISSN 2349-4476

[8] L.Sharma, A. Gera," A Survey of Recommendation System: Research Challenges", Volume4Issue5- May 2013.

[9] J.Beel, B. Gipp, S.Langer, and CorinnaBreitinger," Research-Paper Recommender Systems: A Literature Survey"

[10]Jiajun.Bu,Xin.Shen,Bin.Xu,chunchen,XiaofeiHe,DengCai," Improving Collaborative Recommendation via User-Item subgroups"in IEEE,2016.

[11] Leonardo Zanetsste, Claudia L.R. Motta, Flávia Maria Santoro, Marcos Elia “A Trust-based Recommender System for Collaborative Networks" 2009 IEEE.

[12] A. Kuepper, "Recommender systems," eCommerce, vol. Chapter 08, p. 13, 2011.

[13] A scalable collaborative filtering algorithm based on localized preference, vol. 1 , 2008. [Online].Available:http://dx.doi.org/10.1109/ICMLC.2008.4620397

[14] Michael J. Pazzani and Daniel Billsus, "Content Based Recommendation System."

[15] Diego Campo, MiquelSonsona, Jose-Miguel Pulido "A hybrid recommender combining user, item and interaction data".

[16] Claypool, M., A. Gokhale, T. Miranda, P. Murnikov, D. Netes, and M. Sartin, "Combining content-based and collaborative filters in an online newspaper". In ACM SIGIR'99, Workshop on Recommender Systems: Algorithms and Evaluation, August 1999.

[17]Rong Hu," Acceptance Issues of Personality-based Recommender Systems", RecSys'09, October 23-25, 2009, New York, New York, USA. ACM 978-1-60558-435-5/09/10 (pp 221-224)

[18]J.A. Konstan, J. Riedl, A. Borchers, and J.L. Herlocker, "RecommenderSystems: A GroupLens Perspective," Proc. Recommender Systems, Papers from 1998 Workshop, Technical Report WS-98-08, 1998

[19]Pasquale Lops, Marco de Gemmis and Giovanni Semeraro," Content-based Recommender Systems: State ofthe Art and Trends",Recommender systems handbook ,,73-105,2011.

[20]Joost de Wit B.Sc, "Evaluating Recommender Systems”TNO Information and Communication Technology,Delft, May 16, 2008

\section{BIOGRAPHIES}

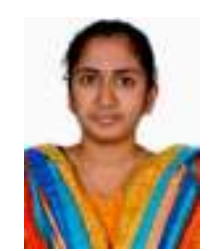

PRIYA Sreceived a BE degree in Computer Science and Engineering from avinashilingam university in 2015. She currently purses ME in the Department of Computer Science and Engineering at the Sri Krishna College of Engineering and Technology, Coimbatore, India. Her research interests include recommendation system in Data Mining. She has presented 2papers in national conference and 1 paper in international conference.He has published 1 paper in international journal.

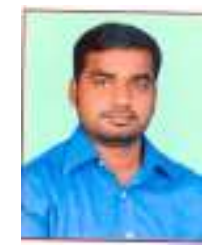

MANSOOR HUSSAIN D is with CSE department in Sri Krishna College of Engineering and Technology, Coimbatore as Assistant professor. He has done his B.E in computer Science and Engineering from University of Madras and M.E. in Computer Science and Engineering from Anna University, Chennai. His research interests include Big Data. He has presented 5 papers in national conference and 2 papers in international conferences. He has published 2 papers in international journal 